\title{
Mechanism of Photoinduced Dihydroazulene Ring-Opening Reaction
}

Abedi, Mostafa; Pápai, Mátyás Imre; Mikkelsen, Kurt V.; Henriksen, Niels Engholm; Møller, Klaus Braagaard

Published in:

Journal of Physical Chemistry Letters

Link to article, DOI:

10.1021/acs.jpclett.9b01522

Publication date:

2019

Document Version

Peer reviewed version

Link back to DTU Orbit

Citation (APA):

Abedi, M., Pápai, M. I., Mikkelsen, K. V., Henriksen, N. E., \& Møller, K. B. (2019). Mechanism of Photoinduced Dihydroazulene Ring-Opening Reaction. Journal of Physical Chemistry Letters, 10, 3944-3949.

https://doi.org/10.1021/acs.jpclett.9b01522

\section{General rights}

Copyright and moral rights for the publications made accessible in the public portal are retained by the authors and/or other copyright owners and it is a condition of accessing publications that users recognise and abide by the legal requirements associated with these rights.

- Users may download and print one copy of any publication from the public portal for the purpose of private study or research.

- You may not further distribute the material or use it for any profit-making activity or commercial gain

- You may freely distribute the URL identifying the publication in the public portal

If you believe that this document breaches copyright please contact us providing details, and we will remove access to the work immediately and investigate your claim 


\title{
On the Mechanism of Photoinduced
}

\section{Dihydroazulene Ring-Opening Reaction}

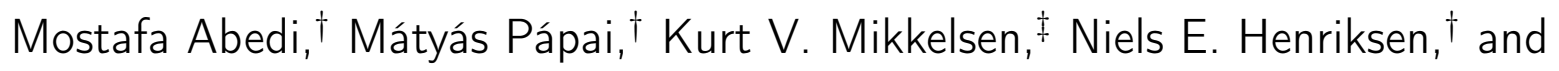 \\ Klaus B. Møller*,† \\ $\dagger$ Department of Chemistry, Technical University of Denmark, DK-2800 Kongens Lyngby, \\ Denmark \\ $\ddagger$ Department of Chemistry, University of Copenhagen, Universitetsparken 5, DK-2100 \\ Copenhagen, Denmark \\ E-mail: kbmo@kemi.dtu.dk
}




\begin{abstract}
The photoinduced ring-opening reaction is a key process in the functioning of dihydroazulene/vinylheptafulvene (DHA/VHF) photoswitches. Over the years, the mechanism of this reaction has been extensively debated. Herein, by means of nonadiabatic trajectory dynamics simulations and quantum chemistry calculations, we present the first detailed and comprehensive investigation on the mechanism of the photoinduced ring-opening reaction of DHA. The results show the crucial role of the excited-state ring planarization process for the bond breaking. Our dynamics simulations show that the DHA ring opening is an ultrafast reaction that does not follow exponential kinetics but exhibits ballistic dynamics. Upon photoexcitation, the planarization occurs within 300-500 fs. This leads to the ring-opening reaction and concurrent decay of the molecule to the ground state within $100 \mathrm{fs}$ through an $\mathrm{S}_{1} \rightarrow \mathrm{S}_{0}$ internal conversion process towards forming the VHF isomer. These results are consistent with previous ultrafast time-resolved experiments and lead to a thorough understanding of the DHA/VHF photoconversion.
\end{abstract}

\title{
TOC Graphic
}

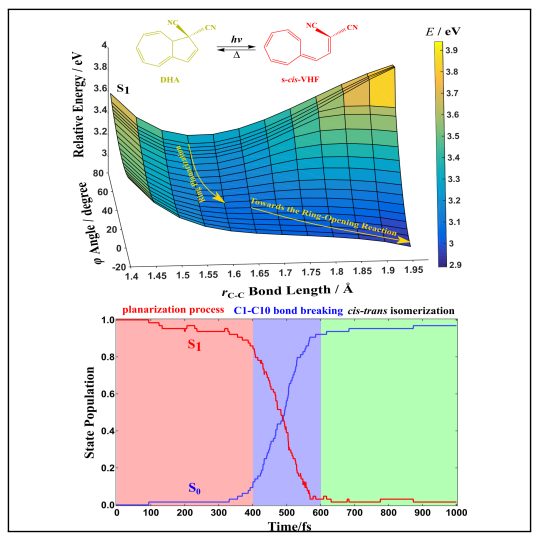


The limited access to fossil fuels makes solar energy conversion and storage one of the great goals and challenges of this century. Among the many existing methods for sunlight conversion like artificial photosynthesis ${ }^{1}$ and photovoltaic devices ${ }^{2}$, to name a few, the strategy for conversion and storage of solar energy into the chemical bonds of metastable photoisomers has attracted great attention. Dihydroazulene (DHA) has been considered for a long time a promising candidate for solar energy harvesting, storage and release. ${ }^{3-9}$ Upon light absorption, the DHA molecule is excited from the electronic ground state $\left(\mathrm{S}_{0}\right)$ to the lowest-lying singlet excited state $\left(\mathrm{S}_{1}\right)$, corresponding to a $\pi \pi^{*}$ transition. The excited molecule undergoes a photoinduced high quantum yield ring-opening reaction, which leads to formation of the metastable s-cis-vinylheptafulvene ( $\mathrm{VHF}$ ) isomer through an $\mathrm{S}_{1} / \mathrm{S}_{0}$ conical intersection (CI). ${ }^{10-13}$ The s-cis-VHF isomer can either undergo a thermal ring-closure reaction with a relatively large energy barrier $\left(18-24 \mathrm{kcal} / \mathrm{mol}^{14,15}\right)$ to recapture the DHA or be transformed to the metastable s-trans-VHF isomer with a energy barrier of $6 \mathrm{kcal} / \mathrm{mol}^{15}$ via a thermal cis-trans isomerization process (Scheme 1).

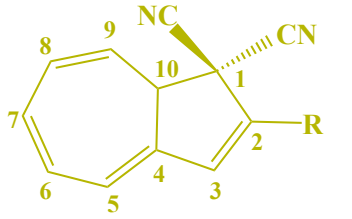

DHA

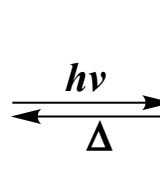

-cis-VHF

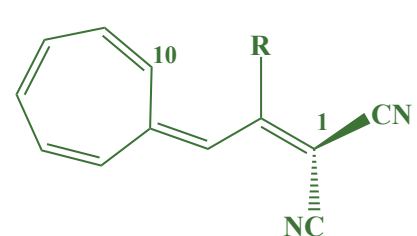

s-trans-VHF

Scheme 1: Schematic representation of the photoinduced DHA ring-opening reaction and thermal VHF cis-trans isomerization. The DFT calculated C1-C10 bond lengths are 1.6 $\AA, 3.3 \AA$ and $4.4 \AA$ for the DHA, cis-VHF and trans-VHF isomers, respectively, at the M06/cc-pVDZ level of theory.

The fast initial step of DHA/VHF photoconversion is the ring-opening reaction. Over the years, this reaction has been experimentally investigated for different DHA derivatives. De Waele et al. ${ }^{12}$ studied the ultrafast dynamics of 1,1-dicyano-2-(4-cyanophenyl)1,8a-dihydroazulene (CN-Ph-DHA; $\mathrm{R}=\mathrm{CN}-\mathrm{Ph}$ in Scheme 1$)$ in methanol using transient absorption spectroscopy and reported time constants of 1.2 ps and 13 ps for the excitedstate ring-opening and $\mathrm{S}_{1} \rightarrow \mathrm{S}_{0}$ internal conversion processes, respectively. Employing the 
same spectroscopic technique, Ern et al. ${ }^{11}$ observed that the whole process including ringopening reaction and formation of ground-state VHF isomer happens in 0.6 ps for 1,2,3,8a,9pentahydrocyclopent[a]azulene-9,9-dicarbonitrile (cp-DHA; R = cp in Scheme 1) in acetone. More recently, the first gas-phase measurement was carried out by Schalk et al. ${ }^{10}$ on the 2-phenyl-1,8adihydroazulene-1,1-dicarbonitrile derivative (Ph-DHA; $\mathrm{R}=\mathrm{Ph}$ in Scheme 1) using time-resolved photoelectron spectroscopy. They reported a time constant of $320 \pm 50$ fs for the ring-opening process. These experimental studies indicate that the ring-opening reaction is strongly dependent on the environment and substitution on the rings. To understand and most importantly control this reaction, it is crucial to get profound knowledge about its mechanism. However, complications and difficulties in the interpretation of ultrafast experiments restrict our current understanding. Theoretical approaches are powerful and complementary tools to extend our knowledge in this regard. In this work, we present the first comprehensive and detailed theoretical investigation on the photoinduced mechanism of the DHA ring-opening reaction. We mention that recently, ring-opening reactions of organic molecules, such as 1,3-cyclohexadiene, have received great attention. ${ }^{16,17}$

Using the criteria for aromaticity, it can be realized that DHA derivatives in the $\mathrm{S}_{0}$ state are not aromatic, however, s-cis-VHF isomers are. This is because in the $\mathrm{S}_{0}$ state DHA is not planar conjugated and thus violates the Hückel rules. For a ring to be planar conjugated, the hybridization of all carbon atoms must be $\mathrm{sp}^{2}$. However, in the $\mathrm{S}_{0}$ state of DHA, the $\mathrm{C} 1$ and $\mathrm{C} 10$ are $\mathrm{sp}^{3}$ hybridized. By excitation of the molecule to the $\mathrm{S}_{1}$ state, an electronic transition occurs from the $\pi$ orbital of the seven-membered ring to a $\pi^{*}$ orbital of the five-membered ring (see Figure S3), which leads to formation of a planar aromatic cycloheptatrienyl cation and increased $\pi$-conjugation, and therefore, stability of the molecule in the excited state. Based on this, we will argue that the ring-opening process occurs through several steps: (i) After excitation from the $\mathrm{S}_{0}$ state, the non-planar sevenmembered ring begins the planarization. (ii) This results in the cleavage of the $\mathrm{C} 1-\mathrm{C} 10$ bond and change of hybridization of the $\mathrm{C} 1$ and $\mathrm{C} 10$ atoms from $\mathrm{sp}^{3}$ to $\mathrm{sp}^{2}$, as well as formation 
of an open-shell diradical intermediate, which has been recently observed experimentally ${ }^{18}$. (iii) The ring-opening reaction takes place and enhances the $\pi$-conjugation system of the molecule to form the metastable VHF isomer. Thus, the ring planarization is hypothesized to be an essential prerequisite for the bond breaking and the ring-opening reaction. To support our arguments, we perform excited-state nonadiabatic trajectory surface hopping (TSH) dynamics simulations, including all nuclear degrees of freedom, using the SHARC 2.0 program package ${ }^{19}$ to explore the dynamical aspects of this reaction, and electronic structure calculations to provide a static 2-dimensional (2D) $\mathrm{S}_{1}$ excited-state potential energy surface (PES). To make the calculations practical, we substitute the phenyl group in the parent molecule (Ph-DHA) with a hydrogen atom to create our model system (H-DHA). We justify the chosen model system by similar vertical excitation energies for the H-DHA and Ph-DHA molecules (with a deviation of around 10\%) leading to the same excited state (see Table S1).
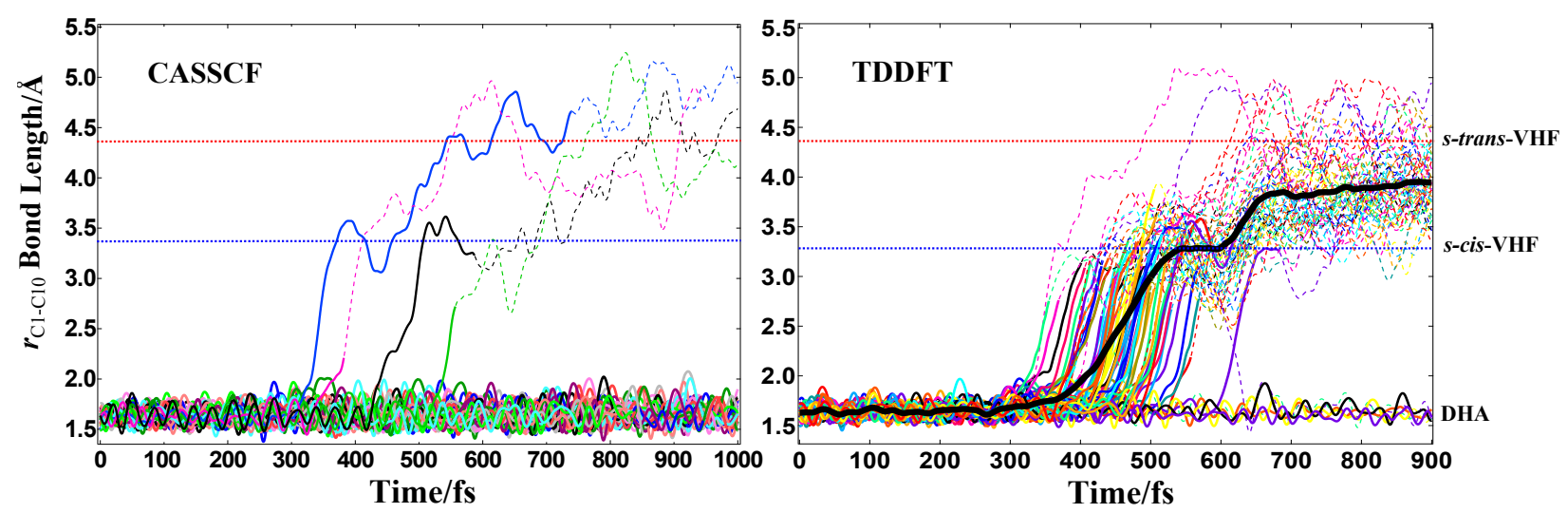

Figure 1: Temporal evolution of the $r_{\mathrm{C} 1-\mathrm{C} 10}$ bond length of the H-DHA obtained from analysis of the trajectories at the SA(4)-CASSCF $(10,10) /$ cc-pVDZ (left) and TD-M06/ccpVDZ (right) levels of theory. The dashed parts of the trajectories show the dynamics in the $\mathrm{S}_{0}$ state. The dotted horizontal lines represent the $r_{\mathrm{C} 1-\mathrm{C} 10}$ bond length from the groundstate CASSCF and DFT optimizations of VHF. The thick black line in the right panel represents $\left\langle r_{\mathrm{C} 1-\mathrm{C} 10}(t)\right\rangle$, obtained by averaging the time-dependent bond length $r_{\mathrm{C} 1-\mathrm{C} 10}(t)$ over all ring-opening trajectories.

We employ the multiconfigurational complete active space self-consistent field (CASSCF) method, which is the most-used approach for describing the $\mathrm{S}_{1} / \mathrm{S}_{0}$ CI. ${ }^{16,17,20,21}$ However, in contrast to the multistate complete active space second-order perturbation (MS-CASPT2) 
and time-dependent density functional theory (TDDFT) methods, the vertical excitation energy obtained from the CASSCF calculation is much higher than the experimental value (Table S1). This is the consequence of wrong excited state ordering due to the lack of dynamic electron correlation in the CASSCF method. The CASSCF excited state ordering at the Franck-Condon (FC) geometry can be seen from Table S2, which shows that the optically allowed state is $S_{3}$ possessing the same character as $S_{1}$ at the TDDFT level. On the other hand, this may not be true when the trajectories leave the FC region and explore other parts of the PESs. For this reason, we carry out excited-state TSH dynamics simulations at the $\operatorname{SA}(4)-\operatorname{CASSCF}(10,10) / \mathrm{cc}-\mathrm{pVDZ}$ level by propagating 50 trajectories up to $1 \mathrm{ps}$. The photoexcitation is described as an instantaneous FC transition where initial conditions are importance-sampled from the Wigner distribution functions ${ }^{22}$ of the ground-state normal modes of DHA (see Supporting Information for the details). Figure 1, left panel, illustrates the time evolution of the $r_{\mathrm{C} 1-\mathrm{C} 10}$ bond length obtained from analysis of the CASSCF propagated trajectories. Unexpectedly only 4 trajectories $(8 \%)$ lead to the ring-opening reaction and internal conversion to the $\mathrm{S}_{0}$ state. These results indeed show the disability of the CASSCF method to quantitatively describe the DHA ring-opening reaction yield and the necessity to include dynamic electron correlation. For the 4 trajectories that do exhibit ring opening, after photoexcitation, we see an "induction time" $23-26$ of 300-500 fs, reflected by the C1-C10 bond oscillation, before initiation of the bond stretching. This is followed by an irreversible ring-opening reaction and formation of the H-VHF isomers via the $\mathrm{S}_{1} \rightarrow \mathrm{S}_{0}$ internal conversion. By taking the point at which the C1-C10 distance stops to oscillate and undergoes an irreversible elongation, we define a bond length of $r_{\mathrm{C} 1-\mathrm{C} 10} \sim 2 \AA$ for the start point of the ring-opening reaction. 


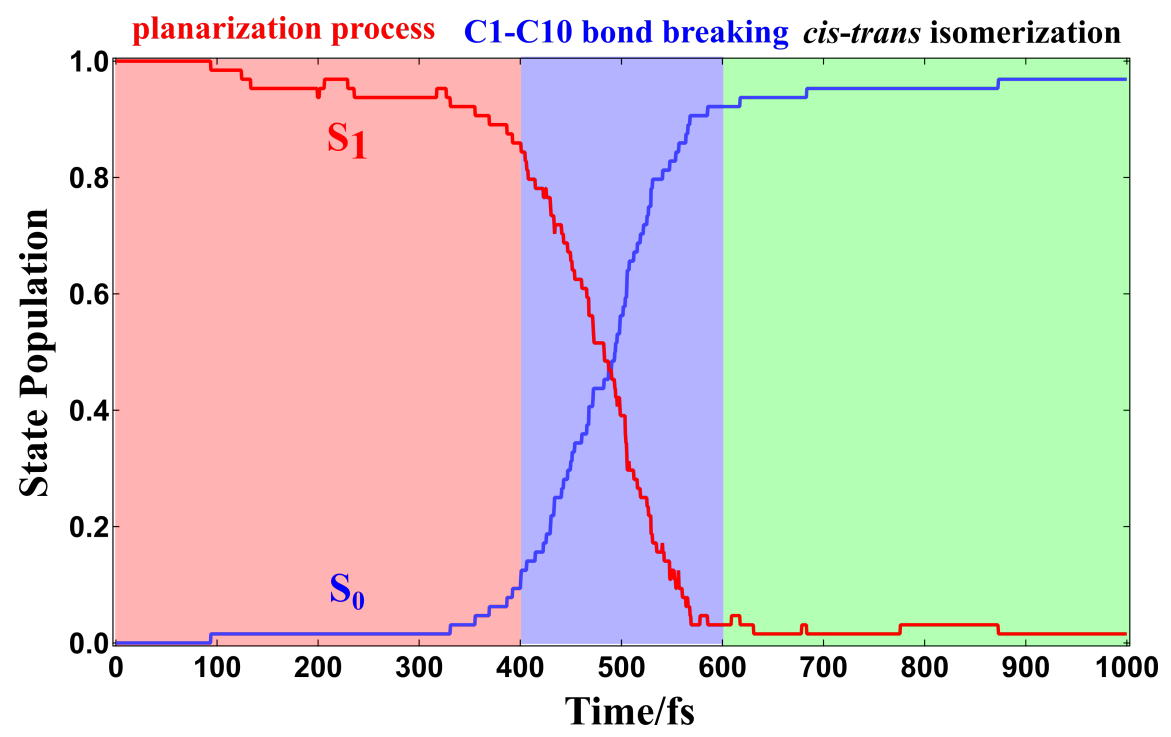

Figure 2: Time evolution of the average electronic state populations along the TDDFT trajectories for the $\mathrm{S}_{0}$ and $\mathrm{S}_{1}$ states. Note that the role of the $\mathrm{S}_{2}$ state in the population decay dynamics is negligible and not shown here.

In order describe the ring-opening reaction yield quantitatively, an alternative choice for the CASSCF method is MS-CASPT2. However, the lack of analytical nuclear gradients in the available TSH codes and expensive computational cost hamper us to perform the dynamics at this level, although we note that there are a few recent nonadiabatic TSH dynamics studies that have applied MS-CASPT2 using a local implementation of analytic energy gradients. ${ }^{27-29}$ Therefore, we make use of the (TD)DFT method as a practical alternative to CASSCF. It is well-known that the TDDFT method fails in the vicinity of the $\mathrm{S}_{1} / \mathrm{S}_{0}$ CI due to the single-reference character of the ground-state wavefunction. ${ }^{20,30} \mathrm{~A}$ well-established strategy ${ }^{31-35}$ for such cases is to analyze the trajectories until the energy gap between $\mathrm{S}_{0}$ and $\mathrm{S}_{1}$ reaches a pre-defined threshold and consider this point as an indication for the $\mathrm{S}_{1} \rightarrow \mathrm{S}_{0}$ internal conversion. Herein, based on the observations from the CASSCF ring-opening trajectories, we apply a forced irreversible hop to the $S_{0}$ state when the energy gap reaches the threshold value and allow the trajectories to continue their exploration into the ground state. Note that the applied strategy of forced hops most likely makes the results biased towards the formation of VHF isomers and thus the obtained quantum yield for the ring-opening 
reaction should be considered as an upper bound. We set $0.15 \mathrm{eV}$ for the threshold guided by the CASSCF simulations and the fact that at this energy gap there is a high probability for the $\mathrm{S}_{1} \rightarrow \mathrm{S}_{0}$ internal conversion. We propagate 64 trajectories at the TD-M06/cc-pVDZ level of theory and analyze the results (see Supporting Information for the computational details). As shown in Figure 1, right panel, in $90 \%$ of the simulated trajectories the irreversible ring-opening reaction occurs, while 2 trajectories undergo the ring-closure and in 4 trajectories, ring opening does not occur and no dissociation is observed in the $\mathrm{S}_{1}$ state. Figure $\mathrm{S} 4$ represents the temporal distribution of the initiation of the ring opening at $r_{\mathrm{C} 1-\mathrm{C} 10} \sim$ $2 \AA$ and subsequent internal conversion, extracted from the TDDFT dynamics simulations. These results show that after photoexcitation within 300-500 fs the C1-C10 bond stretching initiates (see Movie S1b), which is in perfect agreement with the timescale obtained from the $4 \mathrm{CASSCF}$ ring-opening trajectories. Thus, despite of wrong description of the CASSCF method in the FC region, the validity of the ring-opening trajectories may be justified by the facts (1) that the predicted timescale for the initiation of the ring opening obtained from the CASSCF results agrees very well with the one extracted from the TDDFT simulations and with the experiment, and (2) that the average hopping geometries in the $\mathrm{S}_{1} / \mathrm{S}_{0}$ crossing seam region obtained are similar from the TDDFT and CASCF trajectories (see Figure S7) - excluding the CASSCF trajectory with the very late hop to $\mathrm{S}_{0}$ at around $750 \mathrm{fs}$ (the blue trajectory in Figure 1). However, this trajectory shows ring-opening dynamics like the others. As seen from the fast expansion of $r_{\mathrm{C} 1-\mathrm{C} 10}$ shown in Figure 1 and Movie $\mathrm{S} 1 \mathrm{~b}$, the ring opening is an ultrafast reaction, happening in $\sim 25 \mathrm{fs}$. For the average trajectory shown as the thick black line in Figure 1, the ring-opening process appears slower due to the variation in the onset of the ring opening. Thus, the ring opening exhibits "ballistic dynamics" 23-26 that does not follow exponential kinetics. This ballistic dynamics can be also seen clearly from Figure 2 that shows $\mathrm{S}_{1} \rightarrow \mathrm{S}_{0}$ population decay dynamics along the TDDFT trajectories for the first picosecond.

We identify from the dynamics (see Movie S1a and the normal mode analysis of all tra- 


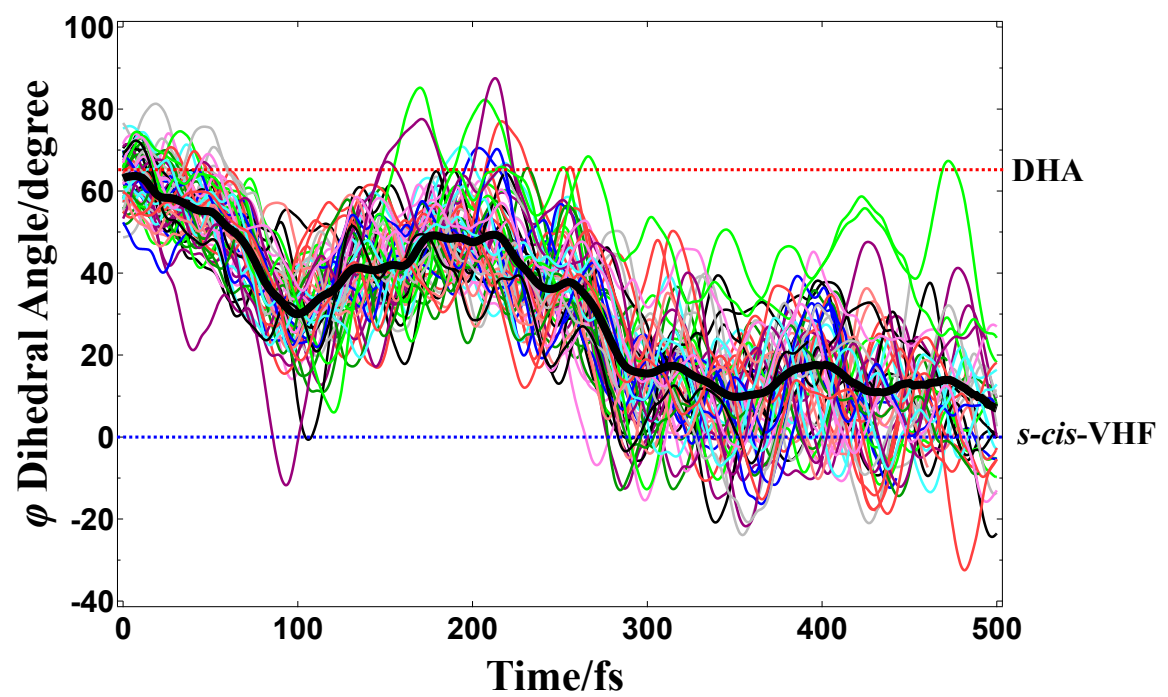

Figure 3: Time evolution of the $\varphi$ dihedral angle of the H-DHA obtained from analysis of the trajectories at the TD-M06/cc-pVDZ level of theory. The thick black line represents $\langle\varphi(t)\rangle$, obtained by averaging the time-dependent dihedral angle $\varphi(t)$ over all trajectories. The dotted horizontal lines represent the $\varphi$ dihedral angle from the ground-state DFT optimization.

jectories as shown in Figures S5 and S6) that the ring planarization occurs prior to the ring opening. To quantitatively characterize this process, we choose the C4-C10-C9-C8 dihedral angle $(\varphi)$ of the seven-membered ring as a descriptor, since this $\varphi$ angle in the equilibrium geometries of the H-DHA and s-cis-H-VHF are $\sim 65^{\circ}$ and $\sim 0^{\circ}$, respectively. Figure 3 illustrates the changes in the $\varphi$ angle along time and clearly shows the ring planarization process occurring prior to the ring-opening reaction. An oscillation with a period of 200 fs $(\sim 170$ $\mathrm{cm}^{-1}$ ) is seen, which can be ascribed to motion of the molecule facilitating the planarization process. This is most clear from $\langle\varphi(t)\rangle$, the time-dependent dihedral angle $\varphi(t)$ averaged over all trajectories shown as the thick black line in Figure 3. De Waele et al. ${ }^{12}$ also identified experimentally four low-frequency $\left(150-330 \mathrm{~cm}^{-1}\right)$ torsional normal vibrational modes for CN-Ph-DHA, which contribute to the planarization and support the ring opening. We note that in addition to the above discussed 200 fs oscillations, we also observe faster large amplitudes motions in Figure 3 related to $\mathrm{C}-\mathrm{C}$ vibrations; however, on average, these fast oscillations cancel out.

After the ring-opening reaction is initiated, the molecule decays very fast to the $\mathrm{S}_{0}$ state 


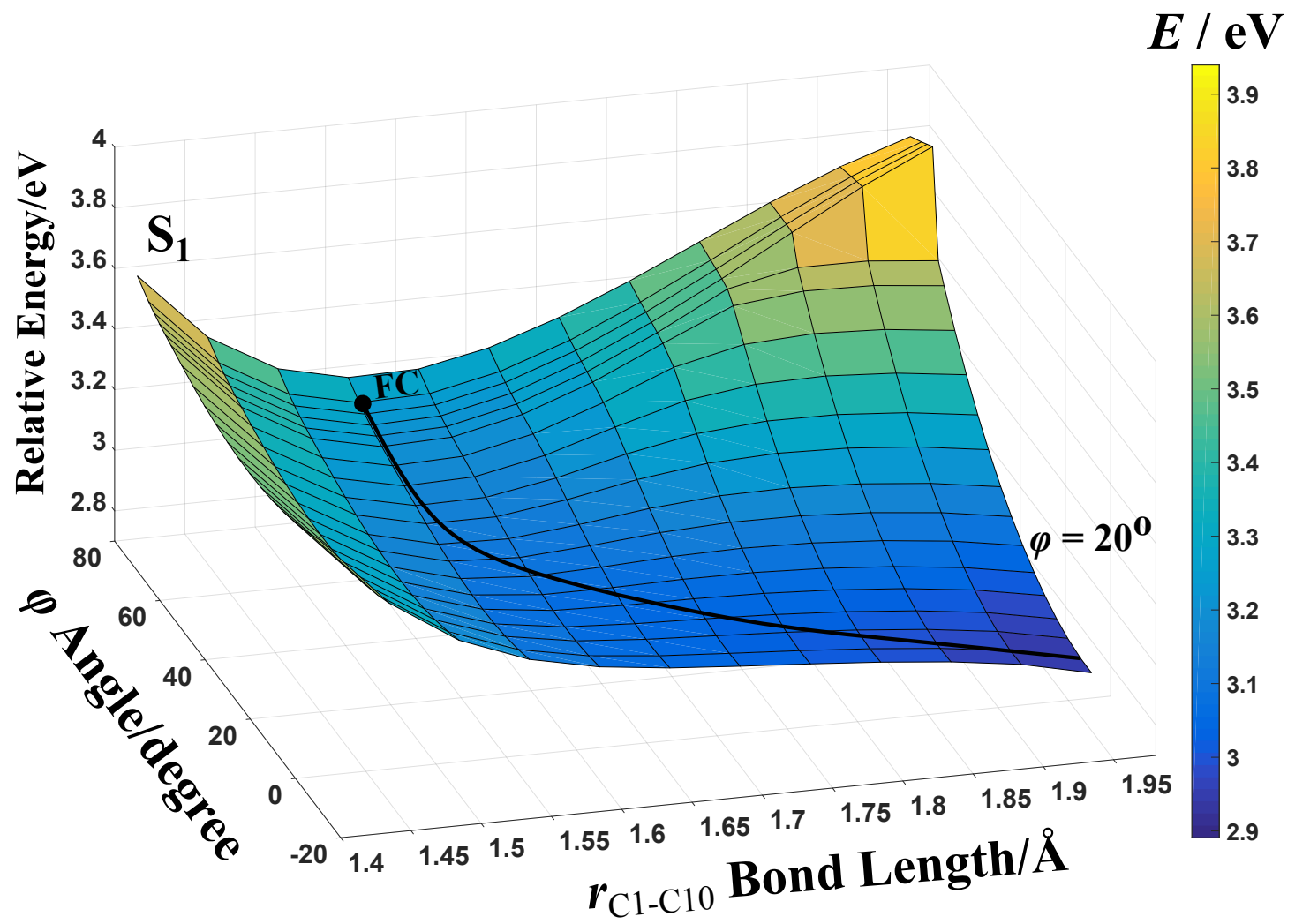

Figure 4: Relaxed 2D PES scan of the $\mathrm{S}_{1}$ state along the $\varphi$ dihedral angle and $r_{\mathrm{C} 1-\mathrm{C} 10}$ bond length computed at the TD-M06/cc-pVDZ level of theory. The black solid line represents the minimum energy path. The energies are given relative to the $\mathrm{S}_{0}$ minimum.

via the $\mathrm{S}_{1} / \mathrm{S}_{0} \mathrm{CI}$, while the $\mathrm{C} 1-\mathrm{C} 10$ bond keeps stretching towards forming the H-VHF isomers. The CASSCF and TDDFT simulations show that the decay process to the $\mathrm{S}_{0}$ state occurs concurrently with the ring opening. Analysis of the TDDFT trajectories indicates that this process happens within $100 \mathrm{fs}$ after initiation of the ring-opening reaction (see Figures 2 and S4). These results show a qualitative agreement between the CASSCF and TDDFT simulations and their consistency with the excited-state experimental observations by Schalk et $a l .{ }^{10}$ and Ern et al. ${ }^{11}$ for Ph-DHA and cp-DHA derivatives, respectively. The analyzed trajectories in the $\mathrm{S}_{0}$ state show a cis-trans VHF interconversion on a sub-ps timescale, as seen from the rapid oscillations of the trajectories within 600-900 fs in Figure 1, in very good agreement with the gas-phase experiment ${ }^{10}$. The reason that this process happens very fast is because the molecule possesses kinetic energy after decay to the $\mathrm{S}_{0}$ and the simulations 


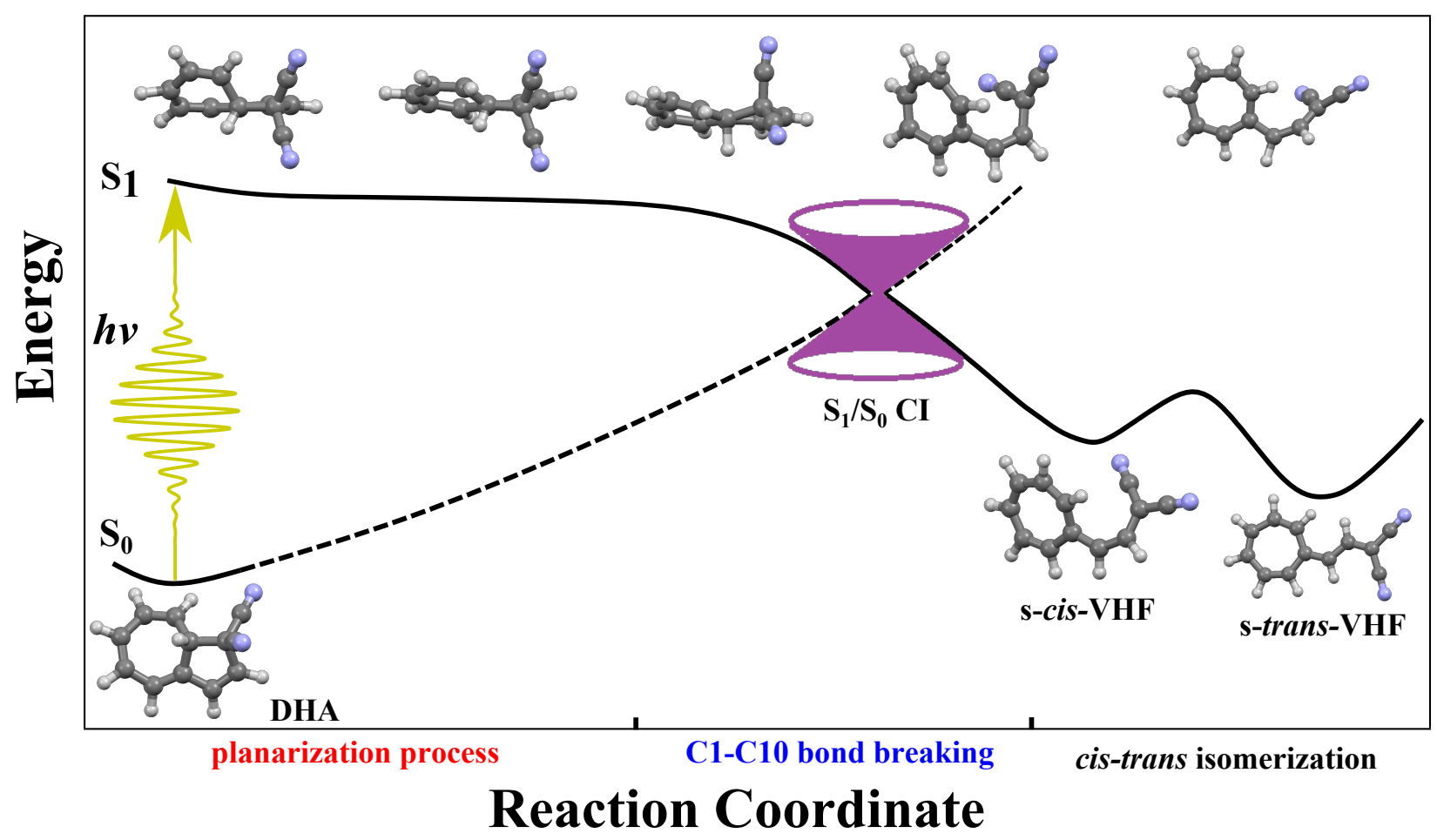

Figure 5: Schematic illustration of the proposed reaction path from the FC region through the $\mathrm{S}_{1} / \mathrm{S}_{0}$ CI towards the cis-trans isomerization process. The reaction coordinate is a combination of the $\varphi$ angle and $r_{\mathrm{C} 1-\mathrm{C} 10}$ bond internal coordinates. The top structures are taken from the dynamics, with top view for the ring planarization and side view for the ring opening. The lines represent the underlying minimum energy path. The bottom DHA and VHF structures are obtained by DFT optimization.

as well as the gas-phase experiment do not enable energy dissipation to the environment (ground-state cooling). We note that since all nuclear degrees of freedom are included in the TSH dynamics, intramolecular vibrational energy redistribution (IVR) can happen. At longer times, this can in principle lead to intramolecular cooling of the dynamics in the reaction coordinate.

Our TSH dynamics simulations indicate that the planarization is a crucial process for the ring-opening reaction. To understand this, we calculated the $S_{1}$ excited-state PES of the H-DHA molecule (see Supporting Information for the computational details). Figure 4 shows the relaxed 2D excited-state PES along the $\varphi$ dihedral angle and $r_{\mathrm{C} 1-\mathrm{C} 10}$ bond length. As seen, due to the existence of high barrier regions $\left(>0.7 \mathrm{eV}\right.$ at $\left.\varphi>20^{\circ}\right)$, the favorable pathway for the bond breaking is only accessible through the ring planarization as shown by 
the black solid line in Figure 4. We point out that in the dynamics, the molecule possessing kinetic energy does not follow the minimum energy path, which can be seen by the C1-C10 bond oscillation in Figure 1. This picture evidences an undeniable role of the planarization process as a bottleneck in the DHA ring-opening reaction. The results of static TDDFT calculations complement and explain the TSH dynamics simulations.

In Figure 5, we present the reaction path including the structural alterations, taken from a representative trajectory (top structures), from the photoexcitation towards the $\mathrm{S}_{1} / \mathrm{S}_{0} \mathrm{CI}$ and cis-trans isomerization. Starting from the FC geometry in the $\mathrm{S}_{1}$ state, the $\varphi$ dihedral angle changes from $\sim 65^{\circ}$ to $\sim 0^{\circ}$ making the structure planar and removing the barrier for the ring-opening reaction. Thereafter, while the molecule keeps the ring planar, the $r_{\mathrm{C} 1-\mathrm{C} 10}$ bond continues to dissociate, and the $\mathrm{S}_{1}$ state approaches the $\mathrm{S}_{0}$ state. The molecule undergoes internal conversion to the $\mathrm{S}_{0}$ state, transforming into a mixture of VHF isomers with cis-trans interconversion occurring on a sub-ps timescale.

\section{Acknowledgement}

This work has been supported by the Danish Council of Independent Research Grant No. 4002-00272 and the Independent Research Fund Denmark Grant No. 8021-00347B. The authors are grateful to Dr. Sebastian Mai from University of Vienna for his useful support and suggestions in the SHARC simulations. MA is thankful to Dr. Elham Azizi for fruitful and helpful discussions.

\section{References}

(1) Styring, S. Artificial photosynthesis for solar fuels. Faraday Discuss. 2012, 155, 357376. 
(2) Grätzel, M. Solar Energy Conversion by Dye-Sensitized Photovoltaic Cells. Inorg. Chem. 2005, 44, 6841-6851.

(3) Cacciarini, M.; Skov, A. B.; Jevric, M.; Hansen, A. S.; Elm, J.; Kjaergaard, H. G.; Mikkelsen, K. V.; Brøndsted Nielsen, M. Towards Solar Energy Storage in the Photochromic Dihydroazulene-Vinylheptafulvene System. Chem. Eur. J. 2015, 21, 74547461.

(4) Skov, A. B.; Broman, S. L.; Gertsen, A. S.; Elm, J.; Jevric, M.; Cacciarini, M.; Kadziola, A.; Mikkelsen, K. V.; Nielsen, M. B. Aromaticity-Controlled Energy Storage Capacity of the Dihydroazulene-Vinylheptafulvene Photochromic System. Chem. Eur. J. 2016, 22, 14567-14575.

(5) Huang, C.; Jevric, M.; Borges, A.; Olsen, S. T.; Hamill, J. M.; Zheng, J.-T.; Yang, Y.; Rudnev, A.; Baghernejad, M.; Broekmann, P. et al. Single-molecule detection of dihydroazulene photo-thermal reaction using break junction technique. Nat. Commun. 2017, 8, 15436 .

(6) Cacciarini, M.; Vlasceanu, A.; Jevric, M.; Nielsen, M. B. An effective trigger for energy release of vinylheptafulvene-based solar heat batteries. Chem. Commun. 2017, 53, $5874-5877$.

(7) Vlasceanu, A.; Frandsen, B. N.; Skov, A. B.; Hansen, A. S.; Rasmussen, M. G.; Kjaergaard, H. G.; Mikkelsen, K. V.; Nielsen, M. B. Photoswitchable Dihydroazulene Macrocycles for Solar Energy Storage: The Effects of Ring Strain. J. Org. Chem. 2017, 82, $10398-10407$.

(8) Vlasceanu, A.; Koerstz, M.; Skov, A. B.; Mikkelsen, K. V.; Nielsen, M. B. Multistate Photoswitches: Macrocyclic Dihydroazulene/Azobenzene Conjugates. Angew. Chem. 2018, 57, 6069-6072. 
(9) Mogensen, J.; Christensen, O.; Kilde, M. D.; Abildgaard, M.; Metz, L.; Kadziola, A.; Jevric, M.; Mikkelsen, K. V.; Nielsen, M. B. Molecular Solar Thermal Energy Storage Systems with Long Discharge Times Based on the Dihydroazulene/Vinylheptafulvene Couple. Eur. J. Org. Chem. 2019, 2019, 1986-1993.

(10) Schalk, O.; Broman, S. L.; Petersen, M. A.; Khakhulin, D. V.; Brogaard, R. Y.; Nielsen, M. B.; Boguslavskiy, A. E.; Stolow, A.; Sølling, T. I. On the Condensed Phase Ring-Closure of Vinylheptafulvalene and Ring-Opening of Gaseous Dihydroazulene. J. Phys. Chem. A 2013, 117, 3340-3347.

(11) Ern, J.; Petermann, M.; Mrozek, T.; Daub, J.; Kuldová, K.; Kryschi, C. Dihydroazulene/vinylheptafulvene photochromism: dynamics of the photochemical ring-opening reaction. Chem. Phys. 2000, 259, 331-337.

(12) Waele, V. D.; Beutter, M.; Schmidhammer, U.; Riedle, E.; Daub, J. Switching dynamics of the photochromic 1,1-dicyano-2-(4-cyanophenyl)-1,8a-dihydroazulene probed by sub30 fs spectroscopy. Chem. Phys. Lett. 2004, 390, 328-334.

(13) Boggio-Pasqua, M.; Bearpark, M. J.; Hunt, P. A.; Robb, M. A. Dihydroazulene/Vinylheptafulvene Photochromism: A Model for One-Way Photochemistry via a Conical Intersection. J. Am. Chem. Soc. 2002, 124, 1456-1470.

(14) Goerner, H.; Fischer, C.; Gierisch, S.; Daub, J. Dihydroazulene/vinylheptafulvene photochromism: effects of substituents, solvent, and temperature in the photorearrangement of dihydroazulenes to vinylheptafulvenes. J. Phys. Chem. 1993, 97, 4110-4117.

(15) Perrier, A.; Maurel, F.; Jacquemin, D. Diarylethene-dihydroazulene multimode photochrome: a theoretical spectroscopic investigation. Phys. Chem. Chem. Phys. 2011, 13, 13791-13799.

(16) Wolf, T. J. A.; Sanchez, D. M.; Yang, J.; Parrish, R. M.; Nunes, J. P. F.; Centurion, M.; Coffee, R.; Cryan, J. P.; Gühr, M.; Hegazy, K. et al. The photochemical ring-opening 
of 1,3-cyclohexadiene imaged by ultrafast electron diffraction. Nat. Chem. 2019, 11, $504-509$.

(17) Lei, Y.; Wu, H.; Zheng, X.; Zhai, G.; Zhu, C. Photo-induced 1,3-cyclohexadiene ring opening reaction: Ab initio on-the-fly nonadiabatic molecular dynamics simulation. $J$. Photochem. Photobiol. 2016, 317, 39-49.

(18) Liepuoniute, I.; Commins, P.; Karothu, D. P.; Schramm, S.; Hara, H.; Naumov, P. Reversible Multicolor Photochromism of Dihydroazulene Crystals. Chem. Eur. J. 2019, 25, 373-378.

(19) Mai, S.; Marquetand, P.; González, L. Nonadiabatic dynamics: The SHARC approach. Wiley Interdiscip. Rev. Comput. Mol. Sci. 2018, 8, e1370.

(20) Gozem, S.; Melaccio, F.; Valentini, A.; Filatov, M.; Huix-Rotllant, M.; Ferré, N.; Frutos, L. M.; Angeli, C.; Krylov, A. I.; Granovsky, A. A. et al. Shape of Multireference, Equation-of-Motion Coupled-Cluster, and Density Functional Theory Potential Energy Surfaces at a Conical Intersection. J. Chem. Theory Comput. 2014, 10, 3074-3084.

(21) Xia, S.-H.; Liu, X.-Y.; Fang, Q.; Cui, G. Excited-State Ring-Opening Mechanism of Cyclic Ketones: A MS-CASPT2//CASSCF Study. J. Phys. Chem. A 2015, 119, 35693576.

(22) Wigner, E. On the Quantum Correction For Thermodynamic Equilibrium. Phys. Rev. 1932, 40, 749-759.

(23) Møller, K. B.; Zewail, A. H. Femtosecond dynamics of transition states: the classical saddle-point barrier reactions. Chem. Phys. Lett. 1998, 295, 1-10.

(24) Møller, K. B.; Zewail, A. H. Kinetics modeling of dynamics: the case of femtosecondactivated direct reactions. Chem. Phys. Lett. 2002, 351, 281-288. 
(25) Kuhlman, T. S.; Pittelkow, M.; Sølling, T. I.; Møller, K. B. Pulling the Levers of Photophysics: How Structure Controls the Rate of Energy Dissipation. Angew. Chem. 2013, 52, 2247-2250.

(26) Kjær, K. S.; Van Driel, T. B.; Harlang, T. C. B.; Kunnus, K.; Biasin, E.; Ledbetter, K.; Hartsock, R. W.; Reinhard, M. E.; Koroidov, S.; Li, L. et al. Finding intersections between electronic excited state potential energy surfaces with simultaneous ultrafast X-ray scattering and spectroscopy. Chem. Sci. 2019, 10, 5749-5760.

(27) Park, J. W.; Shiozaki, T. On-the-Fly CASPT2 Surface-Hopping Dynamics. J. Chem. Theory Comput. 2017, 13, 3676-3683.

(28) Heindl, M.; González, L. A XMS-CASPT2 non-adiabatic dynamics study on pyrrole. Comput. Theor. Chem. 2019, 1155, 38-46.

(29) Polyak, I.; Hutton, L.; Crespo-Otero, R.; Barbatti, M.; Knowles, P. J. Ultrafast Photoinduced Dynamics of 1,3-Cyclohexadiene Using XMS-CASPT2 Surface Hopping. J. Chem. Theory Comput. 2019, DOI:10.1021/acs.jctc.9b00396.

(30) Levine, B. G.; Ko, C.; Quenneville, J.; Martínez, T. J. Conical intersections and double excitations in time-dependent density functional theory. Mol. Phys. 2006, 104, 10391051.

(31) Tapavicza, E.; Tavernelli, I.; Rothlisberger, U.; Filippi, C.; Casida, M. E. Mixed timedependent density-functional theory/classical trajectory surface hopping study of oxirane photochemistry. J. Chem. Phys. 2008, 129, 124108.

(32) Barbatti, M. Photorelaxation Induced by Water-Chromophore Electron Transfer. J. Am. Chem. Soc. 2014, 136, 10246-10249.

(33) Plasser, F.; Crespo-Otero, R.; Pederzoli, M.; Pittner, J.; Lischka, H.; Barbatti, M. 
Surface Hopping Dynamics with Correlated Single-Reference Methods: 9H-Adenine as a Case Study. J. Chem. Theory Comput. 2014, 10, 1395-1405.

(34) Fazzi, D.; Barbatti, M.; Thiel, W. Unveiling the Role of Hot Charge-Transfer States in Molecular Aggregates via Nonadiabatic Dynamics. J. Am. Chem. Soc. 2016, 138, $4502-4511$.

(35) Pang, X.; Jiang, C.; Xie, W.; Domcke, W. Photoinduced electron-driven proton transfer from water to an N-heterocyclic chromophore: nonadiabatic dynamics studies for pyridine-water clusters. Phys. Chem. Chem. Phys. 2019, DOI: 10.1039/C8CP07015F. 